balance. On the contrary, all the patients are still on Milvane. In conclusion, the anticonceptyional treatment with Milvane is not hithetto resulted to be able to worsen the metabolic balance in diabetic patients. Further studies are required during more prolonged treatment to draw any definitive conclusions.

137. Predictive value of the active renin assay for the early diagnosis of ectopic pregnancy

L. Cedard, Th.M. Mignot, K. Meunier, A. Maria ${ }^{1}$, A Guichard, P. Boyer, J.R. Zorn

U. 166 INSERM and Maternité Baudelocque, Paris; ${ }^{1}$ Hôpital Villeneuve-St-Georges, France

The increasing frequency of ectopic pregnancy and the need of its early diagnosis have focused our interest on the research of biochemical markers. We have previously studied the profile of hCG, progesterone, and renin in fertile cycles after IVF-ET or GIFT and shown that his last parameter did not increase in ectopic pregnancies contrary to evolutive or abortive intrauterine pregnancies. After having established the hormonal value in the plasma of 99 spontaneous ongoing pregnancies between the 4th and 10th week of amenorrhea we have compared them to the values observed in 21 ectopic and 20 abortive pregnancies. The hCG levels are significantly higher in ongoing pregnancies but there is no statistically significant difference between abortive and ectopic gestation. The progesterone levels are significantly lower in ectopic pregnancies than in intrauterine gestation but are not really discriminant. The best parameter appears to be low active renin immunoradiometric assay (Pasteur Diagnostic). The difference between ectopic pregnancy and evolutive or abortive intrauterine gestations is highly significant, the highest value corresponding to the 25 th pecentile of normal values. The association of low hCG $(<15000 \mathrm{UI} / \mathrm{ml})$ and low active renin $(\leq$ $30 \mathrm{ng} / \mathrm{ml}$ ) seems to be highly predictive of the ectopic pregnancy. The renin angiotensin system has an important role in the process of implantation, but an early signal could also be transmitted by the implanted embryo to the corpus luteum.

\section{Session 24. Reproductive endocrinology}

138. The effect of polychlorinated Biphenyls (PCB) on estradiol synthesis of human cumulus cells

K. van der Ven, H. van der Ven, A. Thiebold, S. Al-Hasani, U. Wagner, K. Diedrich, and D. Krebs

Department of Obstetrics and Gynaecology, University of Bonn, Bonn, Fed. Rep. of Germany

Chlorinated hydrocarbons, E.G. PCBs, DDT, HCB, can be found in various body tissues and fluids. In human follicular fluid $(n=10)$ we could determine the following concentrations of various PCB congeners (Ballschmitter $n^{\circ} 15,118,138,153,169$, 180) on estradiol synthesis of human cumulus cells was investigated. Cumulus cells were collected in an IVF programme and cultured in a modified Ham's F10 medium supplemented with $5 \%$ fetal calf serum and $50 \mathrm{ng} / \mathrm{ml} 17 \beta$ testosterone (cell concentration: $25000 / \mathrm{ml}$. The PCB congeners were added in concentrations between 0.1 and $1000 \mathrm{ng} / \mathrm{ml}$. The concentration of estradiol was determined in the cell free supernatants after a 24 hour culture period. All PCB congeners induced a dose dependent reduction of estradiol synthesis by human cumulus cells. The various PCB congeners showed different biological activities in regard to the inhibitory effect on estradiol secretion. A decrease of estradiol secretion could already be observed in PCB concentrations that correspond to the average load of human follicular fluid.

139. Endocrine and immunological chances in women with chronic wood preservative exposition

I. Gerhard, M. Derner, B. Runnebaum

Department of Gynaecological Endocrinology, Women's Hospital, University of Heidelberg, Heidelberg, F.R.G.

The influence of environmental pollution on human reproductive performance is unknown. The toxicity of organochlorine compounds (OC) was recongized following accidental poisoning. In animal studies $O C$ administration resulted in a reduced number of pregnancies, and increased number of stillbirths. In the subsequent generation reduced fertility and chances in the menstrual cycle were noted. Wood preservatives containing various toxic $\mathrm{OC}$ are frequently used in industrialized states. Therefore it was the aim of this study to measure the pesticides pentachlorophenol (PCP) and gammahexachlorocyclohexane (Lindane, L) in women with hormonal disorders. In 22 out of 90 women examined elevated OC concentrations were found by capillary chromatography and masspectroscopy. PCP serum levels above $25 \mu \mathrm{g} / \mathrm{l}$ were judged elevated, as were L blood levels above $100 \mathrm{ng} / \mathrm{l}$. Elevated concentrations were found in 8 fertile women (6 of them with repeated abortions, aged 26-33 years), 8 women (aged 16 - 35 years) with menstrual disorders and androgenisation symptoms, and 6 women (aged 42 - 56 years) with climacteric symptoms. The source of emmission was identified in the residence of each patient, the duration of exposure ranged from 2 to 20 years. 16 women presented with adrenocortical malfunction ( 7 with hyperandrogenemia and 9 with partial adrenocorticoinsufficiency), 15 with immunological disorders, 6 with thyroid gland dysfunction (1 with hyper- and 5 with hypothyroidism) and 3 with luteal phase insufficiency. After elemination of the causative noxae and immunologic stimulation, blood $\mathrm{OC}$ concentrations decreased and the patients' physical conditions improved. menstruation normalized and, in case of conception, pregnancies resulted in healthy babies. On the basis of our data OC influence various hormonal systems including the adrenal and thyroid glands and reduce cellular immunity. Therefore an exact evaluation of environmental pollutions should be included in the endocrinological work-up of the patients with hormonal disorders and reproductive failure.

140. Atrial natriuretic peptide (ANP) and progesterone in ovarian follicluar fluid

E.A.P. Steegers, M. van Heeswijk, J.M.G. Hollanders, H.W. Jongsma, and P.R. Hein

Department of Obstetrics and Gynaecology, University Hospital Nijmegen, The Netherlands 
Synthesis and scretion of ANP is not confined to the heart, but has been shown to be present in other tissues too. ANP is known to affect steroidgenesis in the ovary.

The aim of our work was, to assess the possibilities that: a the human ovary is a source of ANP secretion as well and b ovarian ANP stimulates progesterone secretion.

We investigated ANP as well as progesterone concentrations in preovulatory ovarian follicular fluid and in plasma from 15 women in an in vitro fertilization programme. In twelve of them, follicular fluid was pooled before analysis; follicular fluid of three women was collected separately per follicle.

In all women detectable ANP immunoreactivity was found in ovarian follicular fluid (range $3.3-60.1 \mathrm{pg} / \mathrm{ml}$ ). Follicular fluid concentrations were low in all but one woman who demonstrated a higher ANP level in follicular fluid than in plasma. Follicular ANP and progesterone concentrations were not significantly correlated. These preliminary results suggest that the ovary could well be a site of ANP secretion. If and how ovarian ANP activity affects progesterone secretion remains as yet unknown.

141. Plasma melatonin levels in the spontaneous female cycle, during the intake of oral contraceptive, under treatment with gonadrotropins, and in early pregnancy

R. Zimmermann, T. Delfs, S. Baars, M. Schuhmacher, and F.A Leidenberger

Institute for Hormone and Fertility Reserach, Hamburg, Fed. Rep. of Germany

We have examined the influence of endogenous and exogenous sex steroid hormones on the melatonin (MT) production pattern in women. Blood samples were drawn at 4 hourly intervals over a period of 24 hours and daily MT production was calculated from the area under the MT concentration time curve (AUC). Eight volunteers were studied on the fourth day of the menstrual cycle, the day before luteininzing hormone (LH) surge, the day of LH surge, and in the midluteal phase. The AUCs were $555.5+/-89.3,642.7+/$ $149.0,486.6+/ 108.5$, and $457.2+/$ o 69.6 units (mean $+/$ - S.E.) respectively (differences n.s.). The intake of oral contraceptives was not accompanied by any significant changes in MT production, neither conceming the time point of intake nor the gestagen content of the preparation. The effect of human menopausal gonadotropin (HMG) and human chorionic gonadotropin (HCG) on MT levels could be studied in 6 women. The MT AUCs for the 3rd day after onset of menstrual bleeding, on the day when estradiol exceeded $600 \mathrm{pg} / \mathrm{ml}$, and 7 days after 10,000 u HCG were $889.4+/-169.2,885+/-152.6$, and 773.5 $+/-220.5$ units respectively (differences n.s.). In addition, the MT levels in the 10th week of pregnancy were ivenstigated in 9 women and the AUC obtained was $741.0+/-110.3$ units. In conclusion, an influence of sex steroid hormones on the pineal activity could not be detected. This however, does not exclude a relevance of MT in human reproduction. Possibly, it could act as a chronobiologic transmitter, thus conditioning, the CNS for optimal reproductive performance.
142. Reduced pulsatile LH secretion in follicular phase and impaired progesterone levels in luteal phase of normally menstruating women

P. Inaudi, M. Germond ${ }^{1}$, V. De Leo, S. Baldini, N. D'Antona Dept. of Obstetrics and Gynecology, University of Siena,

${ }^{1}$ Department of Obstetrics and Gynecology, CHUV, CH-1011 Lausanne, Switzerland

Since luteal cells originate from the granulosa-theca cell complex and the increase of granulosa cell number is a gonadotropin-related mechanism, the hypothalamic control of gonadotropin release during follicular phase (FP) seems important for a valid corpus luteum development.

We studied 6 women of 25-37 years and a cycle length of $30+1-2$ days, showing progesterone $(P)$ levels $<20 \mathrm{nmol} / /$ - group 1) and 6 healthy age-matched women taken as control ( $P>20$ nmol/1 - group 2); blood samples were taken every 10 min for 6 hours from 8 a.m. in early FP (days 3-6) for LH determination (IFMA method, Cyberfluor, POL YMED, FI, Italy). Furthermore a basal blood sample was obtained every 2-3 days throughout luteal phase (LP) for P evaluation (Sorin Kit, Saluggia, VC, Italy). The characterization of the pulsatile LH patterns was performed by DETECT programme (D. Rodbard, NIH, USA) using a false peak detection level of $1 \%$.

A significant reduction of both basal levels and frequency of the pulsatile LH secretion was found in group 1; a secretory event happens every $120 \mathrm{~min}$ or more (50-60 $\mathrm{min}$ in the control group) with adequate amplitude. Across the LP the P levels in group 1 are generally lower than $20 \mathrm{nmol} / \mathrm{l}$, with a range of $4.5-7.4$ $\mathrm{nmol} / 1 \mathrm{l}(24.6-46.8 \mathrm{nmol} / \mathrm{l}$ in group 2$)$.

The present results show that low P levels during LP can be associated with a decreased LH pulse frequency in follicular phase suggesting that the timing of $\mathrm{LH}$ pituitary discharge could play an important role for an adequate development of corpus luteum.

143. Impaired corpus luteum function associated with mid-luteal long acting GnRH-analogue administration

A. Golan, A. Herman, R. Ron-El, Y. Soffer, H. Nachum, and E. Caspi

IVF unit, Department of Obstetrics and Gyaecology, Assaf Harofe Medical Centre, Tel Aviv University, Israel

Nine spontaneous pregnancies associated with mid-luteal administration of long acting GnRH-analog are reported. A definite impaired function of the corpus luteum was demonstrated by the low progesterone and estradiol levels despite rising levels of HCG. Three of these early pregnancies were supplemented and 2 ended as pre-clinical abortions. The other 6 were treated with progesterone and 3 of them with additional estradiol valerate. Of these, one ended as a blighted ovum and the rest uneventfully at term. It seems that the occurrence of a spontaneous pregnancy in cycles in which GnRH analog is given in the luteal phase are not a rare phenomenon. It is even possible that the transient stimulatory phase following its administration may facilitate implantation. Early diagnosis and close hormonal monitoring are strongly recommended for evaluation of the corpus luteum function and adequate supplementation. 
144. Triggering ovulation by progesterone in healthy subjects

S. Zalanyi jr.

A. Szent-Györgyi University School of Medicine, Szeged, Hungary

Five regularly menstruating healthy subjects were monitored by ultrasound folliculometry and daily blood sampling during the follicular phase of a control and a treatment cycle. These procedures were repeated a second time daily when the follicle reached $16 \mathrm{~mm}$ diameter until ovulation was confirmed. In the treatment cycle progesterone (P) $10 \mathrm{mg}$ in oil was injected i.m. to induce ovulation when the follicle attained $18 \mathrm{~mm}$ which was about two days prior to the expected day of ovulation. The administration of $P$ was guided by ultrasound folliculometry alone. Blood samples were processed for estimation of serum estradiol (E), P and luteinizing hormone (LH) later on. All the subjects ovulated both in control and treatment cycles, as demonstrated by folliculometry, LH peak and luteal phase $\mathrm{P}$ values. $\mathrm{LH}$ peak followed $\mathrm{P}$ adminstration uniformly by 24 hours and was advanced by 36 hours on average (range 12-48 hours) shortening the follicular phase from 12.8 to 11.3 days. E2 peak values were lowered but LH peak and luteal phase P levels remained the same in the treatment cycle. These observations provide evidence that exogenous $\mathrm{P}$ may initiate ovulation in healthy subjects mimicking all events (drop of $E_{2}, \mathrm{LH}$ peak and follicular rupture) thought to be characteristic of the physiological process itself. They further substantiate the theory that $\mathrm{P}$ may be (one of) the trigger(s) of ovulation.

\section{Opioid antagonism prevents the placebo} response in pre-menstrual syndrome

J.H. Schagen van Leeuwen, E.R. te Velde, H.P.F. Koppeschaar, J.H.H. Thijssen, W.J. Kop, J.M. van Ree, and A.A. Haspels University Hospital Utrecht, The Netherlands

A double blind placebo controlled study with nalmefen, an oral opioid antagonist, was perfomed to assess the role of endogenous opioids in the pre-menstrual syndrome (PMS). Twenty-three women, whose diagnosis of PMS was confirmed by longitudinal prospective monitoring of complaints, participated. Nalmefen $(n=12)$ and placebo group $(n=11)$, were comparable for age, body mass index, length of cycle, parity, marital and employment status. Placebo was administered throughout the menstrual cycle, while nalmefen group started active medication on day 12, after taking placebo in the follicular phase. in the placbo group, the highly significant fluctuation of PMS symptoms observed during the selection $(1,2)$ and pre-treatment $(3,4)$ cycles, completely disappeared in the "treatment" $(5,6)$ cycles. In contrast, symptoms remained cyclic during nalmefene treatment. Concomitantly, to acquire indirect evidence of central opioid activity on luteinizing hormone releasing hormone (LHRH), and to assess the effect of nalmefene on menstrual cycle related hormones, plasma levels of $\beta$-endorphin, LH, FSH, oestradiol and progesterone $(\mathrm{P})$ were measured on day 12,22 , and 26 of cycle 3,5, and 6. Both in the placebo and nalmefen group, a significant increase in $\beta$-endorphin occurred, $(p<0.03)$. In the placebo group LH, FSH, oestradiol and P decreased, whereas following nalfene these levels increased. Mid-luteal $P$ levels differed significantly: $p<0.01$.
These results suggest that the placebo response in PMS is mediated by endogenous opioids. Antagonism of these opioids prevents the placebo induced amelioration in PMS. The involvement of central opioid activity, through LHRH, is reflected by an increase in sex hormones with nalmefene, while on placebo the hormone profile attenuated? Also the increase in peripheral $\beta$-endorphin is difficult to explain other than as a central event. Placebos, when perceived as remedy, apparently have pharmacological properties.

\section{Session 25. Progress in prolactin-lowering therapy (Symposium Sandoz)}

146. Hyperprolactinemia treated with modified release bromocriptine (parlodel SRO)

A. Luizzi, D. Dallabonzana, R. Cozzi, S. Strada, G. Oppizzi, P.G. Chiodini, and I. Lancranjan 1

Division of Endocrinology, Ospedale Niguarda, Milan, Italy;

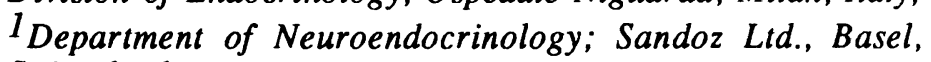
Switzerland

Parlodel SRO (slow release oral) is a new formulation of bromocriptine that allows to obtain retarded peak plasma levels and a significantly longer half life than the standard preparation of Parlodel; these pharmacokinetcs prompted us to evaluate its effectiveness during acute and chronic administration in patients with pathological hyperprolactinemia (PHP).

Acute studies. A single dose of $5 \mathrm{mg}$ SRO given to 13 patients with PHP determined a significant reduction of PRL levels from the 2nd up to the 24th hour (Baseline $1033 \pm 346$ $\mathrm{ng} / \mathrm{ml}$; mean \pm SE; nadir: $193 \pm 3 \mathrm{ng} / \mathrm{ml} ; 24$ hours: $359 \pm 81$ $\mathrm{ng} / \mathrm{ml}$ ).

Chronic studies in a double blind randomized study of one month performed in 20 patients, 5 or $10 \mathrm{mg}$ of SRO given as a single daily adminstration proved as effective in lowering PRL levels as the equivalent doses of Parlodel given b.i.d. In more prolonged studies SRO, given in a single daily adminstration at doses ranging from 5 to $10 \mathrm{mg} /$ day for a mean period of 7.5 months (range 0.5 - 18) to 8 untreated PHP patients, determined a reduction of PRL levels (from $1352.2 \pm 901 \mathrm{ng} / \mathrm{ml}$ to $40 \pm 18.9$ $\mathrm{ng} / \mathrm{ml} ; \mathrm{p}<0.01$ ), a recovery of the gonadal function in $3 / 8$ and a shrinkage of the macroadenoma in 4/5. In other 14 PHP patients shifted to SRO from a previous treatment with equivalent doses of Parlodel, PRL levels showed a further significant decrease from $56.4 \pm 8.6 \mathrm{ng} / \mathrm{ml}$ to $24.7 \pm 5.5 \mathrm{ng} / \mathrm{ml}(\mathrm{p}<0.01)$. Gonadal function, impaired before treatment in all these patients, had normalized in 12/14 during Parlodel and also in the two remaining patients during SRO. Eight out of 10 macroadenomas shrank during Parlodel; a frurther reduction of the tumor occurred in 3 of them during SRO. Only 2/27 patients complained of side effects during SRO treatment.

We conclude that SRO is a further advancement in the pharmacological approach by dopamine agonists to PHP as it allows goor clinical results by a once a day adminstration.

(Study supported by Special Programme of the National Research Council "Oncology", Grant $n^{\circ} 88.00723 .44$ ) 